\title{
Numerical Study of Synchronization in the Kuramoto Model with Finite Inertia
}

\author{
Chulho CHOI \\ Department of Physics and Astronomy, Seoul National University, Seoul 151-747 \\ Meesoon $\mathrm{HA}^{*}$ \\ Graduate School of Education and Department of Physics Education, Chosun University, Gwangju 501-759
}

(Received 10 July 2012 : revised 31 July 2012 : accepted 30 August 2012)

\begin{abstract}
We numerically investigate the role of inertia in the synchronization of globally coupled oscillators in the context of the nature of the synchronization transition. Using the modified Kuramoto model with an inertial term studied by Tanaka et. al., [Phys. Rev. Lett. 78, 2104 (1997)], we measure various physical quantities and determine the threshold of the coupling strength for various values of the magnitude of the inertia that each oscillator possesses. In particular, we employ a conventional analysis of finite-size effects as well as a hysteresis curve analysis. Finally, we argue for the possibility of continuous phase transitions even in the presence of inertia, for which the validity of Binder cumulant's dip and the hysteresis curve are discussed as signatures of a discontinuous transition.
\end{abstract}

PACS numbers: 05.45.Xt, 89.75.-k, 05.45.-a

Keywords: Synchronization, Kuramoto model, Inertia, Binder cumulant's dip, Hysteresis curve

\section{INTRODUCTION}

Since Kuramoto introduced a mathematical model of coupled nonlinear limit-cycle oscillators [1] by refining the earlier Winfree's model [2] to be more mathematically tractable, it has become a minimal model for collective synchronization phenomena in real systems ranging from physics to biology. The original Kuramoto model (KM) is very simple but exhibits lots of rich behaviors including a dynamic phase transition, where all the oscillators' phases are tuned by the coupling strength against the diversity of natural frequencies, and eventually reach a phase-locked state (frequency entrainment) including in-phase synchronization with exactly the same value (see [3] for detailed discussions).

Up to now, lots of phase models have been proposed to describe the dynamic behavior of large populations of nonlinear coupled oscillators. Furthermore, it has also been widely discussed that the nature of synchronization

*E-mail: msha@chosun.ac.kr transitions for the continuum version of the original KM and its variants is subject to the shapes of the natural frequency distribution function as well as the sequence generation methods of natural frequencies [4,5].

Consider KM oscillators with finite inertia as follows: Let $\phi_{i}=\phi_{i}(t)$ be the phase of the $i$-th oscillator and $m$ denote the strength of uniform inertia for each oscillator. Then, the nonlinear dynamics of $\phi_{i}$ is governed by the following second-order ordinary differential equations:

$$
m \ddot{\phi}_{i}+\dot{\phi}_{i}=\omega_{i}+\frac{K}{N} \sum_{j=1}^{N} \sin \left(\phi_{j}-\phi_{i}\right),
$$

which are subject to random initial phase-frequency configurations at $t=0$ for $i=1,2, \ldots, N$. Without loss of generality, the average of natural frequencies sets to be zero:

$$
\omega_{c} \equiv \frac{1}{N} \sum_{i=1}^{N} \omega_{i}=0 .
$$

This KM variant with inertia was first introduced by Ermentrout [6] as a phenomenological model to explain 


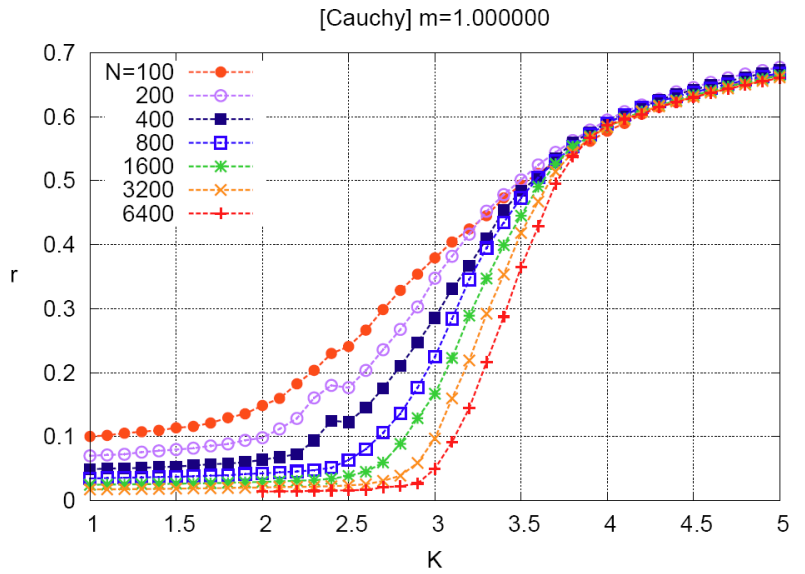

[Cauchy] $m=0.100000$

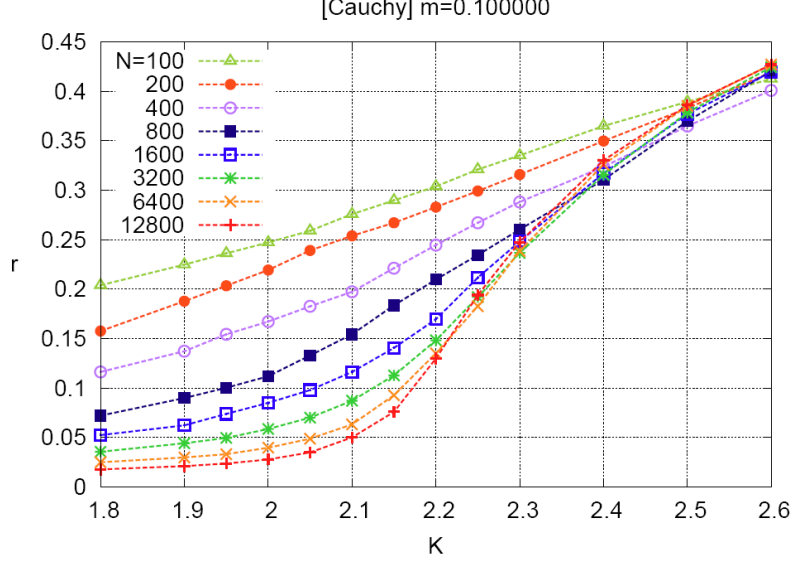

Fig. 1. (Color online) The order parameter $r$ versus the coupling strength $K: \mathrm{m}=1.0$ (left) and $\mathrm{m}=0.1$ (right), respectively, for various system sizes $N$ with $g_{\mathrm{L}}(\omega)$ (Cauchy/Lorentzian) with $\omega_{0}=0$ and $d=1$. Even for $m=1.0$, it seems no jump at a discontinuous transition.

the slow synchronization of certain biological systems, e.g., fireflies of the Pteroptyx malaccae, and later on, it has been used to describe various nonlinearly coupled dynamical systems [7]. Equation (1) can be interpreted as some mechanical analog in the context of the equation of motion: $m \ddot{\phi}_{i}$ (inertia), $\dot{\phi}_{i}$ (damping) and $\omega_{i}$ (driving torque), respectively.

In this report, we numerically investigate the relevance of inertia in synchronization in the context of the issue addressed by Tanaka and coworkers [8] that the modified KM with finite inertia exhibits a first-order (discontinuous) phase transition based on discontinuous jumps and hysteresis curves of the order parameter, in contrast to a second-order (continuous) phase transition found in the original KM. We here test the conventional analysis of finite-size effects as $m \rightarrow 0$ besides the hysteresis analysis of the order parameter.
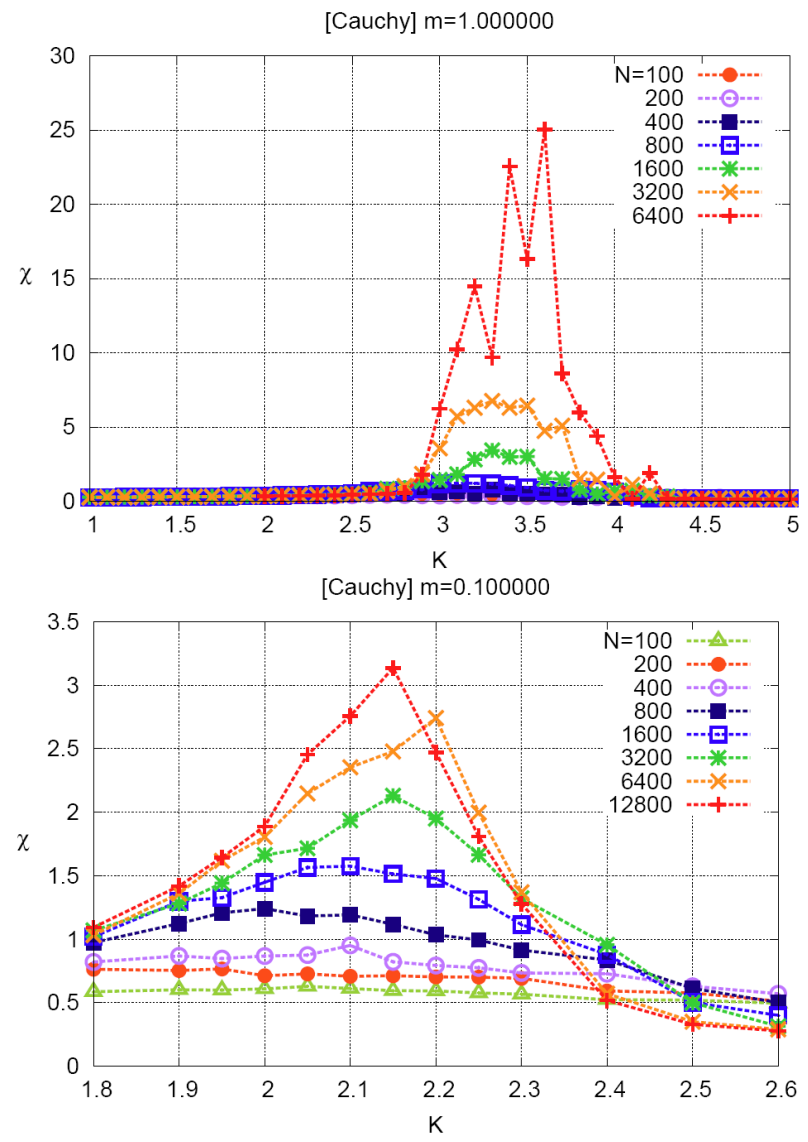

Fig. 2. (Color online) The susceptibility $\chi_{2}^{(1)}$ versus $K$, where we use the same setup as Fig. 1, where the peak of $\chi_{2}^{(1)}$ indicates the location/neighborhood of synchronization threshold.

Based on various properties of the order parameter, we propose comprehensive criteria in determining the nature of phase transitions with either the dips or the crossings of the Binder cumulant (BC) near the threshold of the coupling strength for various system sizes in the presence of different measurements due to anomalous sample-tosample fluctuations. This is attributed to the quenched disorder average of natural frequencies, first discussed by Hong and coworkers in the original KM [9] without inertia. Our BC analysis with finite inertia are compared to the hysteresis curve analysis [8].

This paper is organized after introduction as follows: In Sec. II, basic physical quantities are briefly reviewed and numerically measured for the given distribution of natural frequencies. Based on the conventional analysis of finite-size effects and the $\mathrm{BC}$ analaysis, we argue the nature of the synchronization transition in the modified KM with finite inertia. Finally, some possible scenarios are summarized in Sec. III with open questions. 

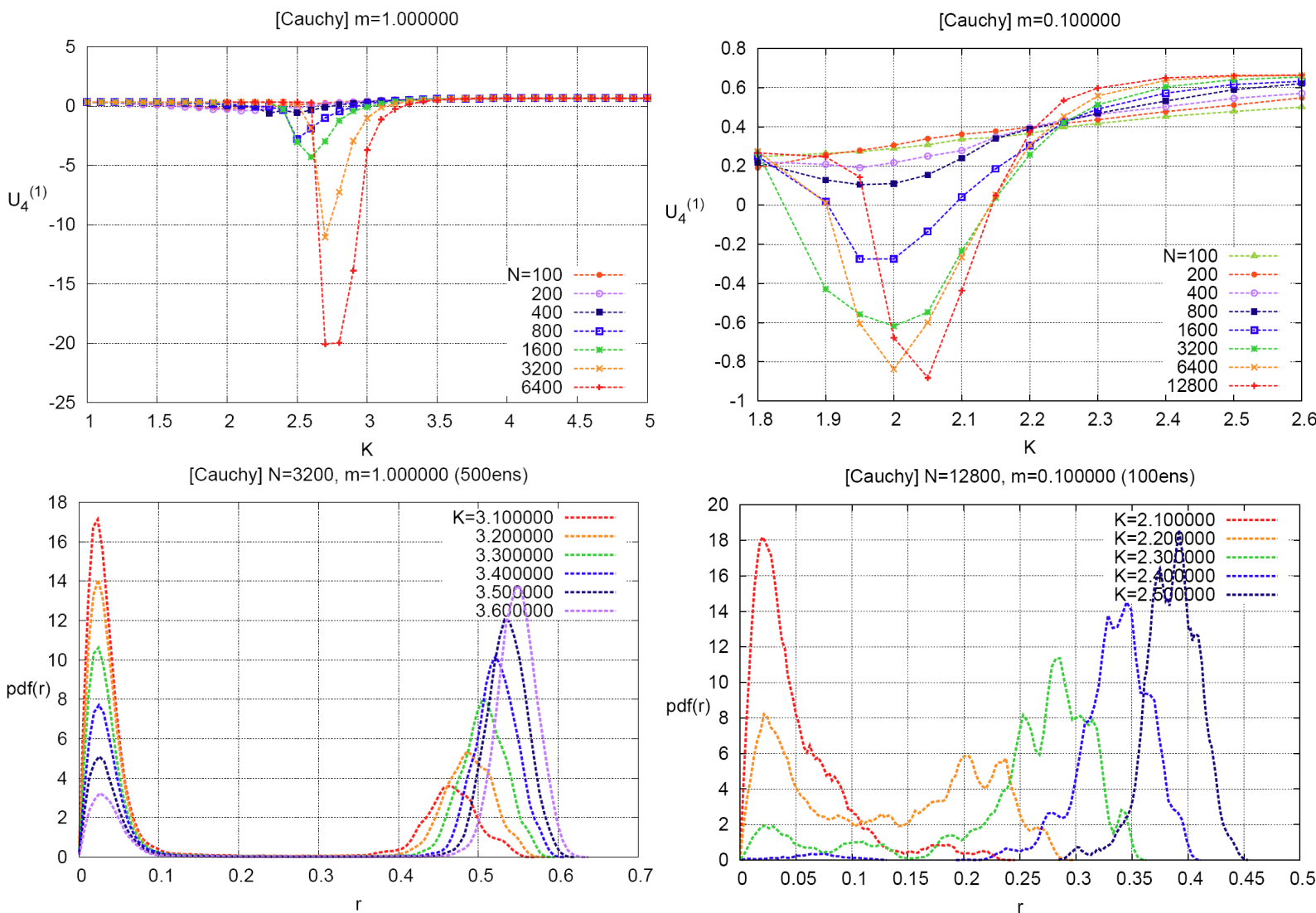

Fig. 3. (Color online) The Binder's cumulant (BC) $U_{4}^{(1)}$ versus $K$ (the upper plots), and the probability distribution function (pdf) of $r$ versus $K$ (the lower plots) with the same setup as Fig. 1. While the BC dips become narrow and deep as $\mathrm{N}$ increases with the clear two-peak pdfs for $m=1.0$, they are relatively wide and shallow with the unclear two-peak pdfs for $m=0.1$. This implies two possible scenarios, the limitation of finite size systems and the evidence of continuous phase transitions for the small values of $m$, respectively.

\section{NUMERICAL RESULTS}

In order to speculate various physical properties of synchronization, we perform the numerical integrations of Eq. (1) by Heun's method, so-called the second order of the Runge-Kutta (RK2) method with two stages [10]. Without loss of generality, the time step $\mathrm{d} t=0.05$ and at least 100 different samples are tested.

The order parameter of synchronization is defined as

$$
r(t) e^{i \theta(t)} \equiv \frac{1}{N} \sum_{j=1}^{N} e^{i \phi_{j}(t)} .
$$

Here we can consider two-types of sampling average, namely the disorder average, [...], over natural frequency sequences and initial configurations, and the temporal average, $\langle\ldots\rangle$, over some period after the system reaches the steady state. While in the steady-state regime the order parameter $r$ yields the same result, regardless of the order of data collections, e.g., $[\langle r\rangle]=\langle[r]\rangle$, other physical quantities can yield different results depending on the definitions, e.g, susceptibility, $\chi_{2}^{(1)} \neq \chi_{2}^{(2)}$, and $\mathrm{BC}, U_{4}^{(1)} \neq U_{4}^{(2)}$.

$$
\chi^{(1)} \equiv N\left[\left\langle r^{2}\right\rangle-\langle r\rangle^{2}\right] ; \quad \chi^{(2)} \equiv N\left(\left[\left\langle r^{2}\right\rangle\right]-[\langle r\rangle]^{2}\right) .
$$

It is well-known that the distribution function shape of natural frequencies determines the transition threshold of the coupling strength and the transition nature. So we specifically choose the single-peak distribution function that exhibits a continuous transition in the original KM in order to focus on the relevance of inertia in the nature of synchronization transitions.

We consider two cases of $g(\omega)$, the Gaussian and Cauchy (Lorentzian):

$g_{\mathrm{G}}(\omega)=\frac{1}{\sqrt{2 \pi \sigma^{2}}} \exp ^{-\frac{\left(\omega-\omega_{0}\right)^{2}}{2 \sigma^{2}}} ; g_{\mathrm{L}}(\omega)=\frac{1}{\pi}\left[\frac{d}{\left(\omega-\omega_{0}\right)^{2}+d^{2}}\right]$. 


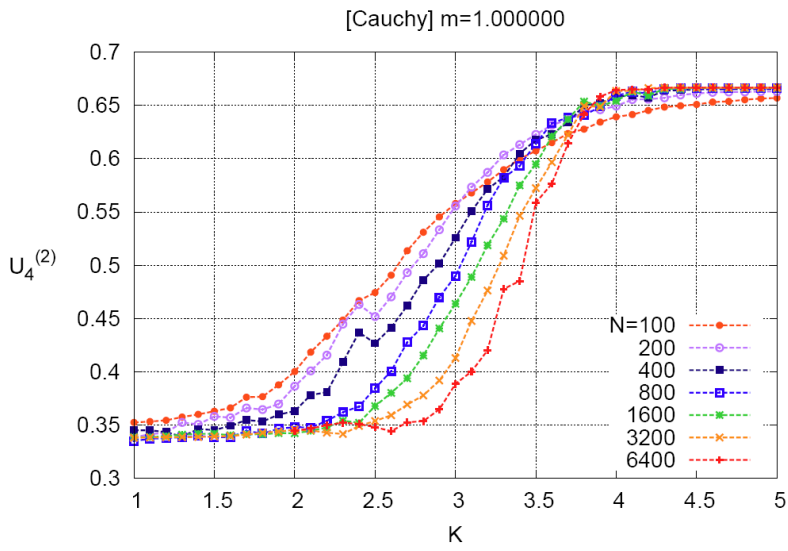

[Cauchy] $m=0.100000$

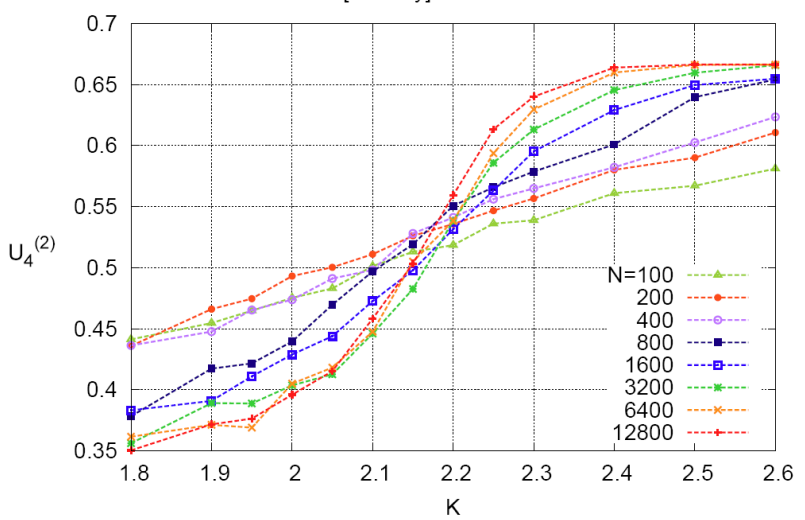

Fig. 4. (Color online) The $\mathrm{BC} U_{4}^{(2)}$ versus $K$, where the same setup is used as Fig. 1. In contrast to Fig. 3, no dips exist. Instead, there are the well-known BC crossings. However, their existence doesn't mean a continuous transition.

Since both functions are characterized by uncorrelated natural frequencies between two oscillators and the finite mean, $\omega_{o}$, so $K_{c}=\frac{2}{\pi g\left(\omega_{0}\right)}$, the data for the case of $g_{\mathrm{L}}(\omega)$ are only presented here.

Once the distribution function of natural frequencies, $g(\omega)$, is chosen, the next step is how to generate the sequence, $\left\{\omega_{i}\right\}$, for finite-size $(N$-oscillator) systems, where $i=1,2, \ldots, N$. One can generate it either stochastically (noisy) or deterministically (noiseless), but the generation method of $\left\{\omega_{i}\right\}$ is directly related to the FSS exponent and the dynamic exponent as reported in $[4,11]$. Moreover, natural frequencies play a role of the quenched disorder in synchronization, the definition of $U_{4}$ should be also carefully discussed as pointed out in [9].

$$
U_{4}^{(1)} \equiv 1-\frac{\left[\left\langle r^{4}\right\rangle\right]}{3\left[\left\langle r^{2}\right\rangle\right]^{2}} ; \quad U_{4}^{(2)} \equiv 1-\left[\frac{\left\langle r^{4}\right\rangle}{3\left\langle r^{2}\right\rangle^{2}}\right] .
$$

In the presence of such a disorder, it is important to check the sample-to-sample fluctuations of physical
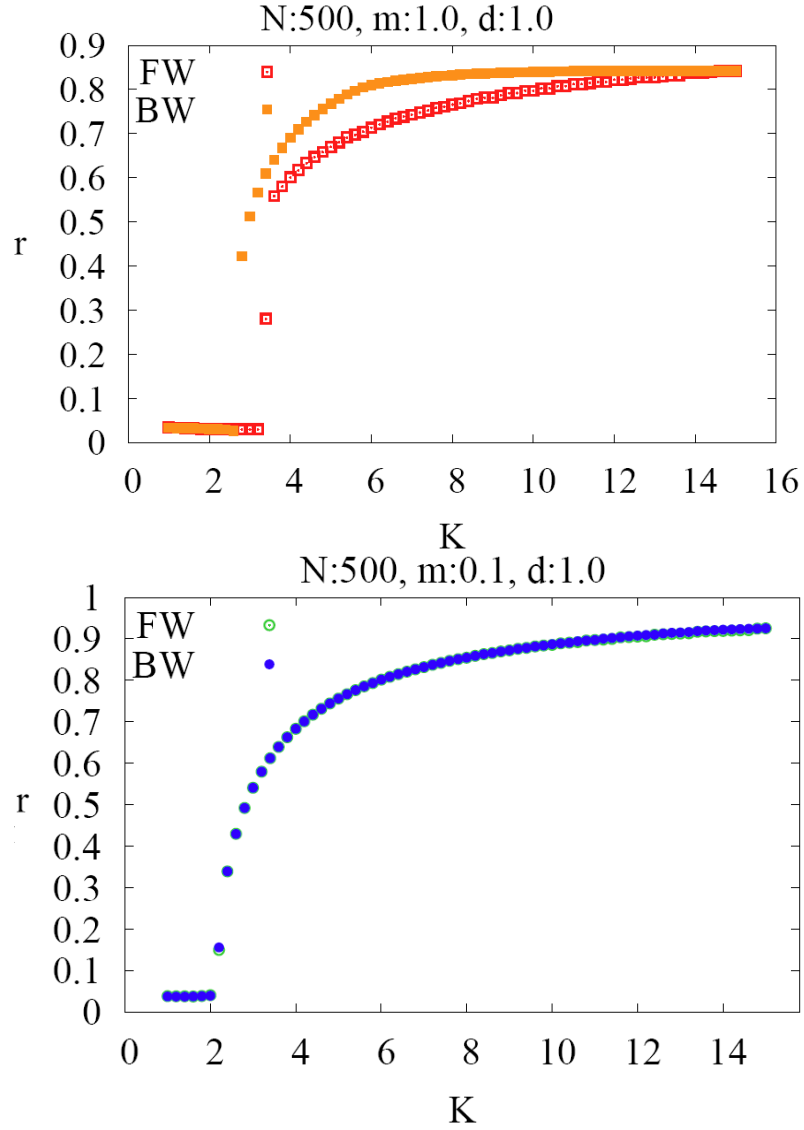

Fig. 5. (Color online) Hysteresis curves of $r$ as $K$ increases (forward, FW) from $r(0)=0$ and decreases from $r(0) \simeq 1$ (backward, BW) for $N=500$ with the same setup as Fig. 1.

quantities, defined as $A_{O} \equiv \frac{\left.[<O\rangle^{2}\right]}{[<O\rangle]^{2}}-1$, where $O$ is any observable physical quantity, e.g., $r$ or $r^{2}$. $A_{O}$ is positive definite, which is supposed to vanish as $N \rightarrow \infty$ if the system is self-averaging; to remains finite if not. For the original Kuramoto model, it has been already reported by Hong and coworkers [9] that $A_{r^{2}}$ saturates to a finite value at criticality due to the relevance of the quenched randomness in natural frequencies. We have observed the similar behavior of $A_{O}$ in the the modified Kuramoto model with finite inertia.

As shown in Figs. 1-5 and stated in the captions, numerical results for $m=1.0$ and $m=0.1$ are quite similar in some aspects, but those are quite different in other aspects. However, with the current resolution of our numerical data, it is not clear to conclude how the BC dips and crossings at the transition threshold are developed in the thermodynamic limit as well as the hysteresis curve of $r$ for the small value of $m$. In other words, we need 
more careful and systematic techniques to analyze finite numerical data obtained from various setup conditions in the context of finite-size scaling and dynamic scaling.

\section{SUMMARY AND REMARK}

In summary, the relevance of inertia in synchronization has been numerically revisited in terms of the modified Kuramoto model with inertia as compared to the ordinary one using the conventional analysis of finite-size effects in the steady-state limit. Our results impose that it is quite difficult how to clarify the nature of synchronization in the presence of inertia even though some criteria of the discontinuous (first-order) phase transition seem exist, such as non-vanishing dips in some specific definition of $\mathrm{BC}$ and hysteresis curves and non-vanishing areas in the thermodynamic limit.

Finally, we have been aware of very slow relaxation in the presence of inertia and the setup-dependent scaling properties through this work. Hence, it is quite crucial to check out if the system reaches its true steady state before discussing the main issue of the transition nature. The dynamic behavior of synchronization near and at the synchronization transition in the modified Kuramoto model is under investigation [11] as compared with the original one.

\section{ACKNOWLEDGEMENTS}

This work is supported by the National Research Foundation of Korea (NRF) grant funded by the Korean Government (MEST) (No. 2011-0011550).

\section{REFERENCES}

[1] Y. Kuramoto, in Proceedings of the International Symposium on Mathematical Problems in Theoretical Physics, edited by H. Araki (Springer-Verlag,
New York, 1975); Y. Kuramoto, Chemical Oscillations, Waves and Turbulence (Springer-Verlag, Berlin, 1984); Y. Kuramoto and I. Nishikawa, J. Stat. Phys. 49, 569 (1987).

[2] A. T. Winfree, J. Theor. Biol. 16, 15 (1967); The Geometry of Biological Time (Springer, New York, 1980).

[3] J. A. Acebrón et. al., Rev. Mod. Phys. 77 135 (2005); B. C. Daniels, Available at http://go. owu.edu/physics/StudentResearch/2005/BryanDan iels/Kuramoto-paper.pdf, 2005.

[4] H. Hong, H. Park, M. Y. Choi, Phys. Rev. E 70, 045204(R) (2005); ibid. 72, 036217 (2005); H. Hong et. al., Phys. Rev. Lett. 99, 184101 (2007); H. Hong, H. Park and L.-H. Tang, Phys. Rev. E 76, 06610 (2007); S.-W. Son and H. Hong, ibid., 81, 061125 (2010); L.-H. Tang, J. Stat. Mech. (2011) P01034.

[5] D. Pazó, Phys. Rev. E 72, 04611 (2005).

[6] G. B. Ermentrout, J. Math. Biol. 29, 571 (1991).

[7] J. A. Acebrón and R. Spigler, Phys. Rev. Lett. 81, 2229 (1998); B. C. Daniels, S. T. Dissanayake and B. R. Trees, Phys. Rev. E. 67, 026216 (2003); K. Park and M. Y. Choi, Phys. Rev. B 56, 387 (1997); K. Wiesenfeld, R. Colet and S. H. Strogatz, Phys. Rev. E 57, 1563 (1988); ibid., Phys. Rev. Lett. 76, 404 (1996); S. Watanabe and J. W. Swift, J. Nonlinear Sci. 7, 503 (1997); S. Watanabe and S. H. Strogatz, Physica D 74, 197 (1994); K. Wiesenfeld and J. W. Swift, Phys. Rev. E 51, 1020 (1995).

[8] H.-A. Tanaka, A. J. Lichtenberg and S. Oishi, Phys. Rev. Lett. 78, 2104 (1995).

[9] H. Hong, H. Park, L.-H. Tang, J. Kor. Phys. Soc., 45, L1885 (2006).

[10] R. L. Burden and J. D. Faires, Numerical Analysis (Brooks/Cole, Pacific Grove, 1997) p.280; Wikipedia, "Runge-Kutta methods", http://en. wikipedia.org/wiki/Runge-Kutta methods.

[11] C. Choi, M. Ha and B. Kahng (in preparation). 